\title{
Considerações Clínicas em Odontogeriatria: plano de tratamento integrado
}

\author{
William Custodio \\ Aluno(a) de Doutorado do Programa de Pós-Graduação \\ em Clínica Odontológica, Área de concentração Prótese \\ Dental, FOP-UNICAMP. \\ Aluna de Mestrado do Programa de Pós-Graduação em \\ Carolina Beraldo Meloto \\ Clínica Odontológica, Área de concentração Prótese \\ Dental, FOP-UNICAMP. \\ Simone Guimarães Farias Gomes \\ Aluno(a) de Doutorado do Programa de Pós-Graduação \\ em Clínica Odontológica, Área de concentração Prótese \\ Dental, FOP-UNICAMP. \\ Professora Titular da Disciplina de Prótese Parcial \\ Removível do Departamento de Prótese e Periodontia, \\ Célia Marisa Rizzatti Barbosa \\ FOP-UNICAMP. (rizzatti@fop.unicamp.br) Fndereço: \\ Célia Marisa Rizzatti Barbosa. Avenida Limeira, n.901, \\ Bairro Areião, Piracicaba-SP. Telefone: (19) 2106-5373 \\ Tipo de Análise do Manuscrito \\ Triple Blind Peer Review \\ Recebido em: Nov/2009 Aprovado em: Nov/2009
}

\section{RESUMO}

As mudanças demográficas revelam um aumento da população idosa cuja saúde oral e geral correlacionam-se diretamente. Esta interação dinâmica assim como as alterações fisiológicas inerentes ao processo de envelhecimento exigem do dentista a expansão de seus conhecimentos para que este adquira uma visão global do idoso a fim de que sejam possíveis a elaboração e execução de um plano de tratamento integrado eficiente que abranja tanto as expectativas quanto as necessidades do paciente odontogeriátrico. A complexidade do indivíduo idoso seja pela maior susceptibilidade às patologias sistêmicas e locais, o uso de polifármacos, a debilidade inerente entre outras características denotam a importância da abordagem sistemática e detalhada acerca do plano de tratamento, evento clínico este muitas vezes menosprezado pelo profissional. Deste modo, o objetivo deste trabalho é discutir as especificidades do plano de tratamento dos pacientes idosos, por meio de uma revisão de literatura científica acerca, de modo que este seja construído abrangendo suas características biopsicossociais de forma clara e objetiva.

Palavras Chave: Plano de tratamento; Idoso; Odontogeriatria

\section{Clinical Considerations in Geriatric Dentistry: integrated treatment plan}

\begin{abstract}
Demographic changes reveal an increase in the elderly population whose oral and overall health are directly correlated. This dynamic interaction as well the physiologic alterations associated to the aging process, requires dentists to expand their expertise to acquire a global view of the elderly subject which became possible the proposition and
\end{abstract}


implementation of an efficient integrated treatment planning that achieve the wishes and real necessities of the odontogeriatric patient. The complexity of the elderly subject due to the higher susceptibility to systematic a local pathologies, usage of polypharmacy and inherent debility, among others factors showed the importance of the systematic and detail approach concerning the treatment planning, a clinical step usually despised. The aim of this study is to discuss, through a literature review, the specificities of the treatment planning of the elderly patients considering its biopsicosocial characteristics in a clear and objective way.

Keywords: Treatment Planning; Elderly; Geriatric Dentistry

\section{Consideraciones clínicas en Odontología Geriátrica: plan integrado de tratamiento de}

\section{RESUIMEN}

Los cambios demográficos muestran un envejecimiento de la población cuya salud bucal y general se correlacionan directamente. Esta interacción dinámica, así como los cambios fisiológicos asociados con el envejecimiento requieren al dentista para ampliar sus conocimientos para que adquiera una visión global de las personas mayores con el fin de ser una posible elaboración e implementación de un plan de tratamiento integrado que incluya la vez eficiente las expectativas sobre las necesidades del paciente odontogeriátrico. La complejidad de los ancianos es el aumento de la susceptibilidad a enfermedades sistémicas y locales, el uso de múltiples drogas, la debilidad inherente entre otras características muestran la importancia de un enfoque sistemático y detallado sobre el plan de tratamiento, caso clínico que se suele pasar por alto por el profesional. Por lo tanto, el objetivo de este trabajo es discutir los detalles del plan de tratamiento de los pacientes de edad avanzada a través de una revisión de la literatura científica acerca de, por lo que se construye cubriendo características biopsicosociales de manera clara y objetiva.

Palabras clave: planificación del tratamiento, Odontología Ancianos, Geriatría

\section{Considérations cliniques en dentisterie gériatrique: plan de traitement intégré}

\section{RÉSUMÉ}

Les changements démographiques montrent une population vieillissante dont la santé bucco-dentaire et générale en corrélation directe. Cette interaction dynamique ainsi que les changements physiologiques associés au vieillissement exigent le dentiste d'élargir leurs connaissances afin qu'il acquiert une vision globale des personnes âgées afin d'être une élaboration possible et la mise en œuvre d'un plan de traitement intégré couvrant à la fois efficace attentes au sujet des besoins du patient. odontogeriátrico La complexité des personnes âgées est l'augmentation de la susceptibilité à des maladies systémiques et locales, l'utilisation de plusieurs médicaments, la faiblesse inhérente entre autres caractéristiques montrent l'importance de l'approche systématique et détaillée sur le plan de traitement, événement clinique qui est souvent négligé par le professionnel. Ainsi, l'objectif de cet article est de discuter des détails du plan de traitement des patients âgés à travers une revue de la littérature scientifique sur, si cela est construite couvrant caractéristiques biopsychosociales clairement et objectivement.

Mots-clés: la planification du traitement, les personnes âgées, dentisterie gériatrique 


\section{Introdução}

A tendência de inversão da pirâmide demográfica, de acordo com dados do Instituto Brasileiro de Geografia e Estatística (IBGE), demonstra o envelhecimento da população, assim como sua feminilização. As projeções sugerem que entre 2000 e 2020 esta faixa populacional constituída por indivíduos acima de 60 anos, aumentará em aproximadamente $6 \%$ e ainda que em 2025 este contingente representará quase $15 \%$ da população total nacional, tornando-nos a sexta maior população idosa do mundo em números absolutos. (1).

Esta mudança de paradigma populacional acarreta, afeta diretamente a classe odontológica, como constatado por estudos $(2,3)$ que evidenciaram que o estereótipo da baixa procura pelos dentistas por parte dos idosos é incorreto e que esta faixa populacional responde por uma quantidade substancial das consultas odontológicas. Consequientemente com o avançar dos anos haverá no Brasil, um maior número de pacientes idosos com suas características biopsicossociais próprias, como a debilidade inerente à idade, maior acometimento por patologias sistêmicas e uso de polifármacos entre outros, buscando por tratamento odontológico, sendo que as necessidades mais freqüientes de tratamento dos idosos estão relacionadas ao edentulismo, à falta de elementos dentários, à cárie dental, abrasões e à doença periodontal (4).

Assim, as mudanças da estrutura etária brasileira e no padrão de saúde bucal, associadas ao fato da constatada falta de preparo dos cuidadores (5) denotam a relevância da retomada de conhecimentos básicos como um adequado diagnóstico do caso clínico afim da elaboração de um plano de tratamento integrado que atinja as expectativas e necessidades reais de modo que a terapêutica clínica transcorra sem intercorrências e de forma eficiente.

Por tratar-se de um passo clínico complexo e de fundamental importância para o sucesso de qualquer terapêutica proposta, este trabalho tem a proposição de discorrer sobre a interferência das condições associadas ao envelhecimento no processo de elaboração do plano de tratamento integrado, revisando a literatura específica.

\section{Plano de Tratamento}

O plano de tratamento é representado por uma fase elaborativa, prévia às diversas etapas curativas (6), ou seja, a estruturação dos passos clínicos até a alta do paciente, construído em função das condições de saúde geral e bucal. Para o delineamento do plano de tratamento, o conhecimento das interações entre as características de saúde geral e as bucais torna-se imprescindível, uma vez que tais fatores são indissociáveis (7).

Para optar pelo tratamento mais adequado a ser estabelecido é necessário que sejam consideradas, individualmente e em conjunto, as variáveis do paciente idoso que englobadas em três grupos constituem as sistêmicas, observadas na anamnese envolvendo os problemas de saúde geral; as bucais, verificadas no exame clínico, radiográfico e modelos de estudo montados em articulador, ou seja, que se referem às condições do sistema estomatognático e, as variáveis psicossociais que abrangem o estado psicológico, a perspectiva de vida do indivíduo, suas expectativas e motivações, além de englobar a disponibilidade financeira (8).

\section{Variáveis Sistêmicas}

No paciente idoso é provável que estas variáveis sejam as mais importantes de ser observadas, uma vez que a partir do conhecimento das mesmas é que podem ser determinadas condutas ou vias alternativas terapêuticas em função da condição de saúde geral do indivíduo. A investigação da condição de saúde geral dos indivíduos idosos deve 
ocorrer durante a anamnese, seja por meio do relato do idoso, de seu cuidador ou mesmo por meio do contato direto médico-dentista.

Um ponto fundamental, não só para a anamnese como também para o desenvolvimento do plano de tratamento é o estabelecimento de uma via de comunicação eficiente. Falhas na comunicação entre o dentista e o paciente geriátrico pode gerar neste, sentimentos de raiva e ansiedade podendo resultar na recusa pelo tratamento ou mesmo na relutância por tratamentos futuros (9). Mudanças de conduta do profissional assim como vias alternativas de comunicação alternativas podem ser úteis no trato com pacientes idosos, como: explicação e conversa sobre o tratamento em ambiente calmo sem barulho externo; estruturação das mensagens cronologicamente; implementação de inquéritos ativos, ou seja, a partir de uma pergunta que remeta o indivíduo a um fato ou conhecimento prévio, este se torna apto a respondê-la; redução da carga de informações por consultas e, finalmente, utilizar recursos como instruções por escrito, imagens explicativas entre outros (10).

Estabelecida a via de comunicação, a anamnese deve transcorrer de forma que sejam obtidos dados claros e objetivos acerca das principais variáveis sistêmicas que possam suscitar alguma diferenciação de tratamento no indivíduo cuja mesma encontra-se alterada.

Um dos principais componentes desta investigação são os problemas cardíacos, segundo Azeredo e colaboradores em 2006 (11) nas grandes capitais brasileiras como São Paulo, Rio de Janeiro e Curitiba, entre outras, mais de $50 \%$ da população acima de 60 anos apresentou diagnóstico clínico de hipertensão arterial, caracterizando esta patologia sistêmica como uma condição comumente encontra nesse grupo populacional. Sendo assim, no plano de tratamento destes indivíduos a fim de evitar eventos como quadros hemorrágicos, acidentes vásculo-cerebrais e mesmo infarto do miocárdio, algumas medidas devem ser adotadas, como a redução da ansiedade/estresse, diminuição do tempo das consultas e ainda a eleição do período matutino para o atendimento dos mesmos (8). Outro fator que deve ser já averiguado é o risco do indivíduo idoso para com os quadros de endocardite bacteriana, no que diz respeito à eleição ou não de profilaxia antibiótica, sendo que atualmente, a recomendação desta terapia é essencialmente dirigida a pacientes com condição cardíaca predisponente de alto risco, na qual incluem-se, por exemplo, portadores de próteses valvares cardíacas e indivíduos com transposição de grandes artérias entre outras (12).

A diabetes, assim como os problemas coronarianos, é uma patologia comum em indivíduos acima de 60 anos e possui correlações com problemas bucais de grande repercussão como os problemas periodontais, como evidenciado em estudos clínicos e epidemiológicos que demonstram a infecção periodontal como um fator de risco para o controle glicêmico em diabetes tipo 2 (13). É importante ressaltar que a grande possibilidade de relatos de glicemia controlada não reais por parte dos pacientes idosos, tornando necessário, portanto o contato direto com o médico do indivíduo, e ainda caso a diabetes não se encontre controlada e o tratamento envolva procedimentos cirúrgicos eletivos a conduta a ser tomada é a não intervenção (8).

Dentre outras variáveis sistêmicas que podem afetar o plano de tratamento como, quadros de confusão mental associados ou não ao Alzheimer; deficiências motoras, por exemplo, decorrentes de artroses que ao comprometer as articulações carpais dificultam a auto higienização, uma terceira variável merece destaque e esta diz respeito ao uso de polifarmácia. Diversos estudos evidenciam o alto consumo de medicamentos pelos idosos, na forma de politerapia ou multimedicamentos, assim como seu uso incorreto, exacerbam o risco de efeitos adversos e interações medicamentosas, passíveis de gerar alterações fisiológicas à estruturas e funções do sistema estomatognático, como a redução do fluxo salivar, que acarreta diversos problemas, desde a xerostomia até a maior incidência de lesões cariosas (14-16) 


\section{Variáveis Bucais}

De acordo com um estudo realizado na cidade de São Paulo, onde o acesso aos serviços sociais, encontra-se teoricamente entre os mais favoráveis de todo o país, a condição de saúde bucal é precária entre os indivíduos idosos. Aos 60 anos ou mais, tanto em idosos funcionalmente ativos assim como em idosos institucionalizados, o índice CPO foi de aproxidamente 30 , ou seja, em cada indivíduo, apenas 2 dois dentes mantinham-se íntegros, sendo que a participação de dentes extraídos variou de 27 a 29 dentes. Em relação ao edentulismo os índices indicam que cerca de 3/4 dos indivíduos perderam todos os dentes e que destes apenas $76 \%$ no domicílio e $30 \%$ nas instituições usavam prótese total superior e inferior e mais agravante ainda, evidenciou-se um elevado percentual de lesões ligadas ao uso de próteses totais tornando evidente a falta de assistência odontológica após a terapêutica reabilitadora. O quadro não é diferente para com as doenças periodontais, sendo que o principal achado foram as bolsas periodontais, caracterizando a necessidade de tratamentos mais complexo e conseqüentemente mais oneroso para mais da $50 \%$ dos idosos examinados (17). Dados este que de acordo com Colussi et al., 2002 (18), são similares para todo território nacional, onde o índice CPOD variou de 26,8 a 31,0 , com grande participação do componente extraído ( $84 \%)$ e alta prevalência de edêntulos (68\%).

De acordo com este panorama os principais pontos que devem ser avaliados, durante o exame clínico extra oral dos idosos, a fim de gerar informações consistentes para a elaboração de um plano de tratamento integrado são as alterações do contorno facial, principalmente acerca da diminuição da dimensão vertical de oclusão; a ocorrência de lesões como quelite angular, ulcerações na língua e mucosa jugal devido a trauma; linha do sorriso; no exame das articulaçôes temporomandibulares a sensibilidade dolorosa à palpação, a presença e tipo de ruídos articulares e ainda a amplitude e trajetória de abertura bucal e, finalmente na avaliação muscular, os índices de sensibilidade principalmente dos grupamentos temporais e masseterinos.

No exame clínico intra-bucal, devem ser averiguados os tecidos moles, devido a alta incidência de afecções como candidose, estomatite protética, leucoplasias e mesmo lesões cancerosas; deve-se proceder a inspeção dos tecidos de suporte como o rebordo residual, no que diz respeito a resiliência da fibromucosa, morfologia horizontal e vertical e ainda os locais de inserção de bridas; a condição dos elementos dentais, principalmente acerca da forma e integridade das coroas clínicas, presença de contatos prematuros, extrusões e inclinações dentais assim como o número e distribuição de dentes remanescentes. Atenção especial deve ser tomada na averiguação de cáries radiculares, uma vez que em idosos o risco para a este tipo de lesão é aumentado devido a maior indecência de retrações gengivais, o uso de dieta cariogênica, diminuição do fluxo salivar e deficiência de higiene bucal (19).

\section{Variáveis Psicossociais}

Mais relevante se tornam os conceitos de saúde e de qualidade de vida com o aumento da população idosa (20). De acordo com a organização Mundial de Saúde (OMS) em 2000, saúde pode ser definida como um estado de completo bem-estar físico, mental e social, e não só a ausência de afecções ou enfermidades (21). A qualidade de vida relacionada à saúde é um conceito dependente da auto-avaliação e relaciona-se à capacidade do indivíduo em interagir no seu grupo social. No idoso, a saúde bucal tem um papel fundamental na autopercepção de saúde e qualidade de vida. Para tanto, entre os principais fatores determinantes temos a capacidade de mastigação, os sintomas de dor e as desordens e doenças bucais (22) sendo que seu comprometimento pode afetar de forma deletéria os níveis nutricionais do indivíduo, seu bem-estar físico e mental, e tornara limitante a convivência e interação social (20). Torna-se necessário, portanto, a busca 
pelas reais expectativas dos indivíduos idoso, afim de que este compreenda, previamente as decisões de tratamento, as limitações de seu caso clínico afim da elaboração de um plano de tratamento que abranja não só as reais necessidades do indivíduo, como também seus anseios na medida do possível.

\section{Divisão do Plano de Tratamento Extenso}

Em pacientes idosos é comum que os casos clínicos envolvam um grande número de procedimentos e áreas, bem como ausência de um ou mais elementos dentários, de maneira que exigem a ordenação adequada dos passos clínicos a serem tomados, demonstrando assim o papel fundamental do plano de tratamento integrado. Este deve, portanto, ser elaborado por sessões de atendimento; abranger todas as fases da terapêtica proposta, conter a descrição de todos os procedimentos que serão realizados e principalmente ser compreendido e aceito pelo paciente idosos ou seu mantenedor.

Devido à alta complexidade, característica comum encontrada entre os pacientes odontogeriátricos, Brunetti e colaboradores em 2002 (8), propuseram a divisão do tratamento do paciente idoso em três fases: tratamento inicial; tratamento final e a manutenção dos resultados obtidos.

O tratamento inicial compreende a intervenção de diversas áreas de conhecimento e visa o sanar o quadro evolutivo de deterioração da saúde bucal. Para que o tratamento seja empregado de forma correta e sem intercorrências faz-se necessário à ordenação dos procedimentos a serem executados da seguinte forma: solução de emergências (relacionados à dor e/ou estética); orientação e motivação do paciente para a higiene bucal; instituição de tratamento periodontal não-cirúrgico; cirurgias (exodontias e mesmo as periodontais); tratamentos endodônticos; restaurações diretas e; próteses provisórias. Uma vez que esta ordem de execução seja seguida, ao final desta fase, o paciente encontrar-se-á com um adequado preparo de boca para receber o tratamento protético reabilitador, necessário em grande parte dos casos clínicos, sendo que se não houver a necessidade de tratamento reabilitador por meio de próteses, esta fase será suficiente para sanar a condição de saúde bucal.

O tratamento final compreende basicamente as reabilitações com próteses, sejam elas, totais ou parciais, removíveis ou fixas. Sendo imprescindível nesta fase a ponderação a respeito da condição de saúde geral e bucal do indivíduo, sua motivação em relação à terapia odontológica, a capacidade técnica e científica do profissional e ainda os custos e viabilidade das modalidades terapêuticas reabilitadoras em função de cada caso. Condutas universais não são possíveis de serem aplicadas aos pacientes odontogeriátricos, contudo, algumas normas gerais podem ser adotadas independentemente do caso, por exemplo, a objetividade em propiciar uma mastigação eficaz, com a formação do bolo alimentar, auxiliando no processo digestivo; uma predileção pela parte funcional do tratamento ao invés da estética; implementação de medidas que facilitem a higienização bucal (próteses removíveis preferíveis neste quesito); a menor oneração possível do tratamento; a ponderação a respeito de tratamentos cirúrgicos extensos e ainda o cuidado do indivíduo com profissionais de áreas afins como fonoaudiologia e fisioterapia.

A manutenção dos resultados obtidos é a fase pós-intervenção, na qual é exigida uma postura ativa do indivíduo em relação à sua saúde bucal. Esta fase compreende, para o cirurgião dentista, basicamente dois pontos a motivação para a rotina doméstica de higiene bucal e das próteses e, o estabelecimento de um esquema regular de consultas para o controle do caso. Sendo que alguns artifícios podem ser utilizados como a orientação da higiene oral por escrito, uso de cartilhas de orientação e ainda cartas de motivação ao paciente em relação a sua saúde bucal e o estabelecimento de consultas semestrais. 


\section{Conclusão}

Da avaliação dos inúmeros fatores que podem afetar o plano de tratamento de indivíduos idosos, deduz-se a maior complexidade dos casos clínicos apresentados por esta população emergente da estrutura demográfica brasileira. Outro fator é a enorme variação individual frente ao delineamento de um plano de tratamento que abranja todas as áreas de conhecimento da odontologia, seja por condições sistêmicas, locais e ou psicossocias, como pela somação e diferentes combinações com que estas variáveis podem acometer o paciente odontogeriátrico. Desta forma, apenas alicerçados em uma adequada anamnese e exame clínico é que se torna possível um correto diagnóstico e plano de tratamento, para que então associados ao conhecimento científico e habilidades técnicas do cirurgião dentista assim como o estabelecimento de um relacionamento interpessoal satisfatório e a motivação do paciente, seja obtida e sustentada o sucesso da terapêtutica proposta.

\section{Referências}

1. Camarano AA. Envelhecimento da população brasileira: uma contribuição demográfica. Texto Para Discussão No 858. IPEA, Rio de Janeiro. 2002.

2. Papas, A. et al. Prevalence and intraoral distribution of coronal and root caries in middle-aged and older adults. Caries Res, 1992. 26(6): p. 459-65.

3. Meskins, L.H.. Dental care for elderly. Journal of American Dental Association, 1992. 123(5): 810.

4. Feijó, E.C. in Menezes, A. e cols. Caminhos do Envelhecer. 1993. Ed Preliminar: UFF/FIOCRUZ/SBGG-RJ, Niterói, RJ, p.101.

5. Saintrain, M.V.L.; Souza E.H.A.; Caldas Junior A.F.. Geriatric dentistry in Brazilian universities. Gerodontol, 2006. 23(4): p. 231-236.

6. Todescan, F.F. et al. Atualização na Clínica Odontológica. 1996. Artes Médicas, $\mathbf{1}^{\mathrm{a}}$ ed. São Paulo.

7. Shinkai, R.S.A \& Del Bel Cury, A.A. O papel da odontologia na equipe interdisciplinar: contribuindo para a atenção integral ao idoso. Cad. Saúde Pública, 200o. 16(4): p. 1099-1109.

8. Brunetti, R.F \& Montenegro, F.L.B.In. Odontogeriatria: Noções de Interesse Clínico. 2002. Artes Médicas, São Paulo.

9. Eli I. Oral psychophysiology. Boca Raton, FL: CRC Press, 1992.

10. Kiyak, H.A. Recent advances in behavioral research in geriatrics dentistry. Gerontol. 1988. 7(1): p. 27-36.

11. Passos, V.M.A.; Assis, T.D.; Barreto, S.M. Hipertensão arterial no Brasil: estimativa de prevalência a partir de estudos de base populacional. Epidemiologia e Serviços de Saúde, Brasil, 2006. 15(1): p. 35-45.

12. Duval, X.; Leport C. Prophylaxis of infective endocarditis: current tendencies, continuing controversies. Lancet Infect Dis, 2008. 8(4): p. 225-32.

13. Taylor, G.W.; Loesche, W.J.; Terpenning, M.S. Impact of oral diseases on systemic health in the elderly: diabetes mellitus and aspiration pneumonia. $J$ Public Health Dent, 2000. 60(4): p. 31320.

14. Fanhani, H.R.; Cuman, R.K.N.; Takemura, S.O.; Seixas, F.A.V.; Andrade, O.G. Consumo de medicamentos por idosos atendidos em um centro de convivência no noroste do Paraná, Brasil. Revista brasileira de Geriatria e Gerontologia, 2007. 10(3): p. 301-314.

15. Linjakumpu, T.; Hartikainen, S.; Klaukka, T.; Veijola, J.; Kivelä, S.L.; Isoaho, R. Use of medications and polypharmacy are increasing among the elderly. $J$ Clin Epidemiol, 2002. 55(8): p. 809-17.

16. Maik, E.; Laskawi, R.; Rohrbach-Volland, S.; Christian, A.; Wolfgang, B. Botulinum Toxin to Reduce Saliva Flow: Selected Indications for Ultrasound-Guided Toxin Application Into Salivary Glands. Laryngoscope, 2002. 112(1): p. 82-6 
17. Rosa, A.G.F.; Fernandez, R.A.C.; Pinto, V.G. \& Ramos, L.R. Condições de saúde bucal em pessoas de 6o anos ou mais no Município de São Paulo (Brasil). Revista de Saúde Pública, 1992. 26(3): p. 155-160.

18. Colussi, C.F. \& Freitas, S.F.T. Aspectos epidemiológicos da saúde bucal do idoso no Brasil. Cad. Saúde Pública, 2002. 18(5): p.1313-1320.

19. Morais, P.M.R.; Hara, A.T.; Serra, M.C. Entendendo a cárie radicular. Rev. Bras. Odont, 1999. 56(5): p. 225-233.

20. Werner, C.W. et al. Odontologia geriátrica. Rev. Fac. Odont Lins, 1998. 11(1): p. 62-70.

21. Organização das Nações Unidas (ONU). Population Fund. Report.20oo.

22. Locker, D. Health outcomes of oral disorders. J Epidemiol, 1995. 24(3): p. S85-S89. 